\title{
Deformation of a two-phase medium due to a long buried strike-slip fault
}

\author{
Sunita Rani ${ }^{1}$, Neeru Bala ${ }^{2 *}$ \\ ${ }^{1}$ Department of Mathematics, Guru Jambheshwar University of Science \& Technology, Hisar, India; \\ s b rani@rediffmail.com \\ ${ }^{2}$ Department of Mathematics, Dayanand College, Hisar, India; ${ }^{*}$ Corresponding Author: neerubala1702@gmail.com
}

Received 6 June 2013; revised 6 July 2013; accepted 13 July 2013

Copyright (C) 2013 Sunita Rani, Neeru Bala. This is an open access article distributed under the Creative Commons Attribution License, which permits unrestricted use, distribution, and reproduction in any medium, provided the original work is properly cited.

\begin{abstract}
The aim of the present paper is to obtain the twodimensional deformation of a two-phase elastic medium consisting of half-spaces of different rigidities in welded contact due to a buried long strike-slip fault. The solution is valid for arbitrary values of the fault-depth and the dip angle. The effect of fault-depth on the displacement and stress fields for different values of dip angle has been studied numerically. It is found that the displacement field varies significantly for a buried fault from the corresponding displacement field for an interface-breaking fault. The contour maps showing the stress field for various dip angles for buried and interface-breaking fault have been plotted. It has been observed that the stress field varies significantly for a buried fault from the corresponding stress field for an interfacebreaking fault.
\end{abstract}

Keywords: Deformation; Two-Phase Elastic Medium; Buried Strike-Slip Fault; Arbitrary Dip

\section{INTRODUCTION}

The elastic residual field due to a strike-slip fault in various Earth models has been calculated by several investigators e.g. [1-12] and others. In [1], the problem of the static deformation of a multilayered half-space by a long strike-slip line dislocation is considered. In [2], the two-dimensional problem of a long displacement dislocation in an isotropic multilayered half-space is studied. In that paper, authors obtained the surface displacement caused by a line source of arbitrary dip. In [3], authors obtained closed-form analytic expressions for the displacements and stresses at any point of either of two homogeneous, isotropic and perfectly elastic half-spaces in welded contact due to a horizontal or a vertical long strike-slip fault. Reference [4] demonstrated the solution for a long strike-slip fault of arbitrary dip, generalizing the work done in [3]. In [5], authors obtained closedform analytic expressions for the problem of a surfacebreaking long strike-slip fault in an elastic layer overlying an elastic half-space. In [6], authors obtained the deformation field at any point of a horizontal orthotropic elastic layer of infinite lateral extent coupling in different ways such as "welded", "smooth-rigid", or "rough-rigid" to a base due to a long blind strike-slip fault. Most of these studies have chosen the interface-breaking fault. The depth of the fault does not occur explicitly in the solution. Therefore, for small dip angles, the fault approaches near the interface and the effect of depth on a fixed dip angle can not be studied independently.

The purpose of present paper is to obtain an analytical solution for the deformation of a long strike-slip fault buried at arbitrary depth located in an elastic, homogeneous, isotropic half-space welded with another elastic, isotropic half-space. The depth occurs explicitly in the solution. Therefore, the effect of the variations in the depth for a fixed dip and vice-versa can be studied directly.

\section{THEORY}

Let the Cartesian co-ordinates be denoted by $\left(x_{1}, x_{2}, x_{3}\right)$ with $x_{3}$-axis vertically downwards. Consider a two-phase elastic medium consisting of halfspaces welded along the plane $x_{3}=0$. The upper halfspace $\left(x_{3}<0\right)$ is called Medium I and the lower halfspace $\left(x_{3}>0\right)$ is called Medium II with rigidities $\mu_{1}$ and $\mu_{2}$, respectively. A long inclined strike-slip fault with strike along $x_{1}$-axis is situated in the lower halfspace. The upper edge of the fault is taken to be at depth $d$ (Figure 1). Superscript (1) denotes quantities related to the upper half-space and superscript (2) denotes those for the lower half-space. 


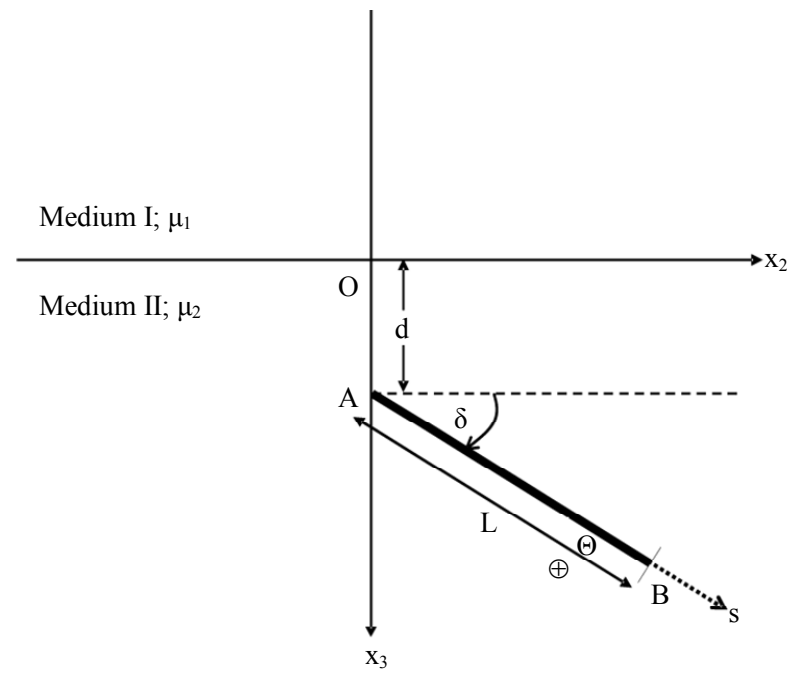

Figure 1. Geometry of a two-phase elastic medium consisting of half-spaces in welded contact with a long strike-slip fault of width $\mathrm{L}$ situated in the lower half-space. $d$ is the depth of upper edge A of the fault, $\delta$ is the dip angle and $s$ is the distance from the upper edge of fault measured in down-dip direction. The displacement discontinuity on the fault is parallel to $x_{1}$ axis. The sign $\oplus$ indicates displacement in the direction of $x_{1-}$ axis and the sign $\Theta$ in the opposite direction.

Under the assumption of antiplane strain case, the displacement components are of the form

$$
u_{1}^{(i)}=u_{1}^{(i)}\left(x_{2}, x_{3}\right), u_{2}^{(i)}=u_{3}^{(i)}=0(i=1,2)
$$

For zero body forces, the equilibrium equations reduces to

$$
\frac{\partial^{2} u_{1}^{(i)}}{\partial x_{2}^{2}}+\frac{\partial^{2} u_{1}^{(i)}}{\partial x_{3}^{2}}=0(i=1,2)
$$

The displacement field due to a long inclined strikeslip line dislocation parallel to $x_{1}$-axis and passing through the point $\left(y_{2}, y_{3}\right)$ in the lower half-space (medium II) is given by [4]:

$$
\begin{aligned}
u_{1}^{(1)}= & \frac{b d s}{\pi(1+\beta) R^{2}}\left[\left(x_{3}-y_{3}\right) \cos \delta-\left(x_{2}-y_{2}\right) \sin \delta\right] \\
u_{1}^{(2)}= & \frac{b d s}{2 \pi}\left[\frac{\left(x_{3}-y_{3}\right) \cos \delta-\left(x_{2}-y_{2}\right) \sin \delta}{R^{2}}\right. \\
& \left.-\frac{1-\beta}{(1+\beta) S^{2}}\left\{\left(x_{3}+y_{3}\right) \cos \delta+\left(x_{2}-y_{2}\right) \sin \delta\right\}\right]
\end{aligned}
$$

where

$$
\begin{aligned}
& b=\text { displacement discontinuity (slip) } \\
& \mathrm{ds}=\text { width of the line dislocation } \\
& \delta=\text { dip angle } \\
& \left(x_{2}, x_{3}\right)=\text { receiver location } \\
& \left(y_{2}, y_{3}\right)=\text { source location }
\end{aligned}
$$

$$
\begin{gathered}
R^{2}=\left(x_{2}-y_{2}\right)^{2}+\left(x_{3}-y_{3}\right)^{2}, S^{2}=\left(x_{2}-y_{2}\right)^{2}+\left(x_{3}+y_{3}\right)^{2} \\
\beta=\mu_{1} / \mu_{2} .
\end{gathered}
$$

We write (Figure 1)

$$
y_{2}=s \cos \delta, y_{3}=d+s \sin \delta
$$

where $d$ is depth of the upper edge A of the fault and $\mathrm{s}$ is the distance from the upper edge of the fault measured in the down-dip direction. Inserting the values of $y_{2}$ and $y_{3}$ from Equation (6) into Equations (3) and (4) and integrating over s between the limits $(0, L)$, we obtain the following expressions for the displacements in the two half-spaces due to an inclined strike-slip fault of finite width $L$ and infinite length:

$$
\begin{aligned}
u_{1}^{(1)}= & \left.\frac{b}{\pi(1+\beta)} \tan ^{-1}\left(\frac{s-x_{2} \cos \delta-X \sin \delta}{X \cos \delta-x_{2} \sin \delta}\right)\right|_{0} ^{L} \\
u_{1}^{(2)}= & \frac{b}{2 \pi} \tan ^{-1}\left(\frac{s-x_{2} \cos \delta-X \sin \delta}{X \cos \delta-x_{2} \sin \delta}\right) \\
& -\left.\frac{1-\beta}{1+\beta} \tan ^{-1}\left(\frac{s-x_{2} \cos \delta+X^{\prime} \sin \delta}{X^{\prime} \cos \delta+x_{2} \sin \delta}\right)\right|_{0} ^{L}
\end{aligned}
$$

where

$$
\left.f(s)\right|_{0} ^{L}=f(L)-f(0)
$$

The non-zero stresses at any point of a two-phase elastic medium are given by

$$
p_{12}^{(i)}=\mu_{i} \frac{\partial u_{1}^{(i)}}{\partial x_{2}}, p_{13}^{(i)}=\mu_{i} \frac{\partial u_{1}^{(i)}}{\partial x_{3}}(i=1,2, \text { no summation over } i)
$$

From Equations (7) and (8) and Equation (10), we get the following expressions for the stresses. For the medium I,

$$
\begin{aligned}
& p_{12}^{(1)}=\left.\frac{\mu_{1} b}{\pi(1+\beta)}\left[\frac{s \sin \delta-X}{R^{2}}\right]\right|_{0} ^{L} \\
& p_{13}^{(1)}=\left.\frac{\mu_{1} b}{\pi(1+\beta)}\left[\frac{x_{2}-s \cos \delta}{R^{2}}\right]\right|_{0} ^{L}
\end{aligned}
$$

and the medium II,

$$
\begin{aligned}
& p_{12}^{(2)}=\left.\frac{\mu_{2} b}{2 \pi}\left[\frac{s \sin \delta-X}{R^{2}}+\left(\frac{1-\beta}{1+\beta}\right) \frac{\left(X^{\prime}+s \sin \delta\right)}{S^{2}}\right]\right|_{0} ^{L} \\
& p_{13}^{(2)}=\left.\frac{\mu_{2} b}{2 \pi}\left[\left(x_{2}-s \cos \delta\right)\left\{\frac{1}{R^{2}}-\left(\frac{1-\beta}{1+\beta}\right) \frac{1}{S^{2}}\right\}\right]\right|_{0} ^{L}
\end{aligned}
$$

where now

$$
\begin{aligned}
& R^{2}=\left(x_{2}-s \cos \delta\right)^{2}+(X-s \sin \delta)^{2} \\
& S^{2}=\left(x_{2}-s \cos \delta\right)^{2}+\left(X^{\prime}+s \sin \delta\right)^{2}
\end{aligned}
$$


Equations (7) and (8) and Equations (11)-(14) give the elastic residual field at any point of two half-spaces due to a long strike-slip fault of finite width dipping at an angle $\delta$ buried at depth $d$. On taking $d=0$, the results for an interface breaking fault located in the lower halfspace welded with another half-space coincide with the corresponding results of [4]. Also on taking $\mu_{1}=0$, which implies $\beta=0$ and $\delta=90^{\circ}$, the results coincide with the corresponding results given by [7] for a uniform half-space due to a vertical strike-slip fault.

\section{NUMERICAL RESULTS}

We have studied the behaviour of the parallel displacements and the stresses numerically. Figure 2(a) shows the parallel displacement $u_{1}^{(1)} / b=u_{1}^{(2)} / b$ at the interface $\left(x_{3}=0\right)$ with the distance from the fault for $\delta=0^{\circ}$ for different values of depth $d$. Figures 2(b)-(d) are for $\delta=15^{\circ}, 30^{\circ}$ and $45^{\circ}$, respectively. We observe that the behaviour of displacement for the interface-breaking fault is altogether different from that for the buried fault. Figures 3(a)-(d) show the variation of parallel displacement $u_{1}^{(2)} / b$ with $x_{2} / L$ at $x_{3}=L$ for three values of depth $d=0, L / 2$ and $2 L$ for $\delta=0^{\circ}, 15^{\circ}, 30^{\circ}$ and $45^{\circ}$. The case $d=0$ corresponds to the interface-breaking fault. For the case $d=L / 2$, observer is below the upper edge of the fault and for $d=2 L$, observer is above the upper edge of the fault.

In all these figures, there is a discontinuity at $x_{2}=\mathrm{X}$ $\cot \delta$. Figures 4(a)-(d) show the variation of $u_{1}^{(1)} / b$ with $\left(x_{2} / L\right)$ for $x_{3}=-L$ for different value of $d$ for $\delta=0^{\circ}, 15^{\circ}, 30^{\circ}$ and $45^{\circ}$ when the observer is in the upper half-space.

The contour maps for the shear stress $p_{12}^{(i)} /\left[\mu_{2} b / L\right]$ have been plotted in Figures 5(a) and (b) for an interface breaking fault located in the lower half-space welded with another half-space for $\delta=0^{\circ}$ and $45^{\circ}$. Solid lines indicate positive values and dashed lines negative values. The values are shown in units of $10^{3} \times p_{12}^{(i)} /\left[\mu_{2} b / L\right]$. Heavy line denotes the fault. The shear stress $p_{12}^{(i)}$ is discontinuous at the interface.

Figures 6(a) and (b) are for the buried strike-slip fault $d=L$ for $\delta=0^{\circ}$ and $45^{\circ}$, respectively. The contour maps for the shear stress $p_{13}^{(i)} /\left[\mu_{2} b / L\right]$ are shown in Figures 7(a) and (b) for interface-breaking fault $d=0$ for $\delta=0^{\circ}$ and $45^{\circ}$. The stress is continuous at the interface. The values are shown in units of $10^{3} \times p_{13}^{(i)} /\left[\mu_{2} b / L\right]$. Figures 8(a) and (b) are for the buried fault $d=L$.

\section{DISCUSSION}

The results presented in this paper are significant

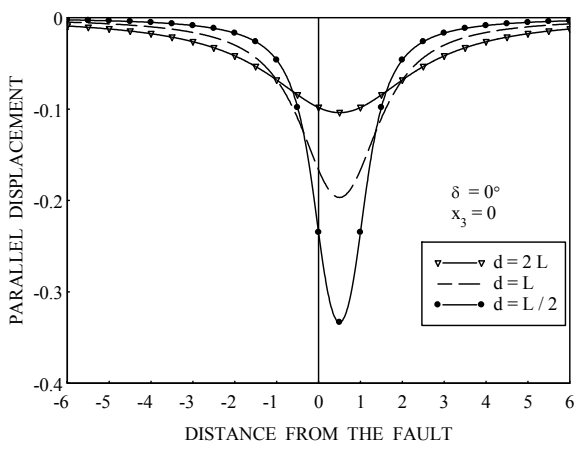

(a)

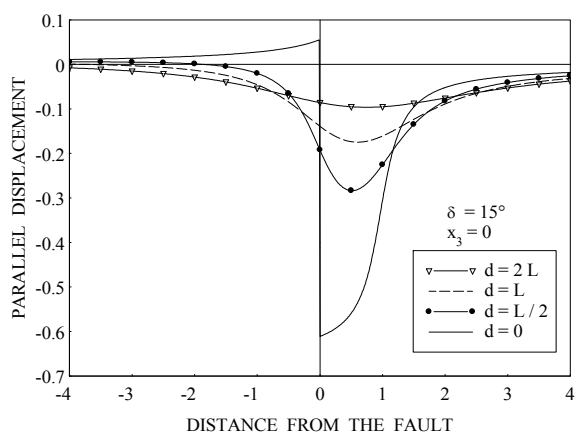

(b)

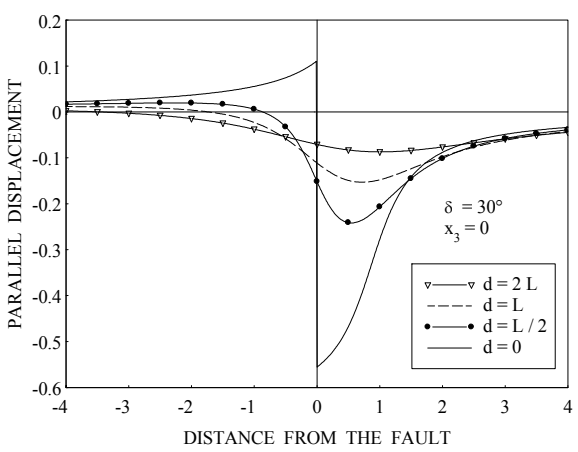

(c)

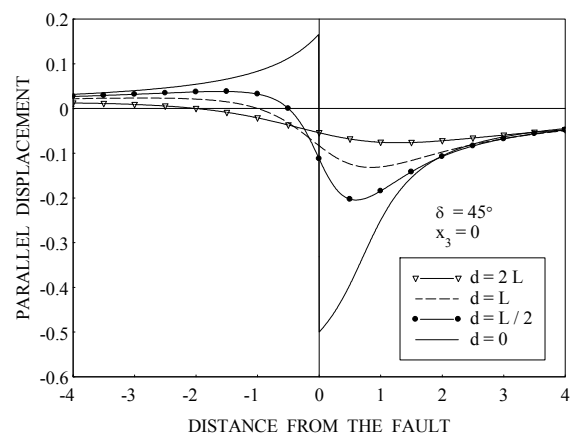

(d)

Figure 2. Variation of the horizontal displacement $u_{1}^{(1)} / b=u_{1}^{(2)} / b$ at the interface with the dimensionless distance from the fault $\left(x_{2} / L\right)$ assuming $\mu_{1} / \mu_{2}=1 / 2$ for various values of depth $\mathrm{d}$ from the upper edge of the fault for (a) $\delta=0^{\circ}$ (b) $\delta=15^{\circ}$ (c) $\delta=30^{\circ}$ (d) $\delta=45^{\circ}$. 


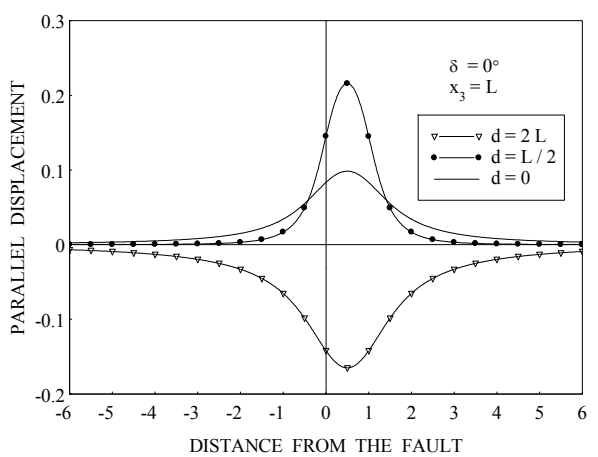

(a)

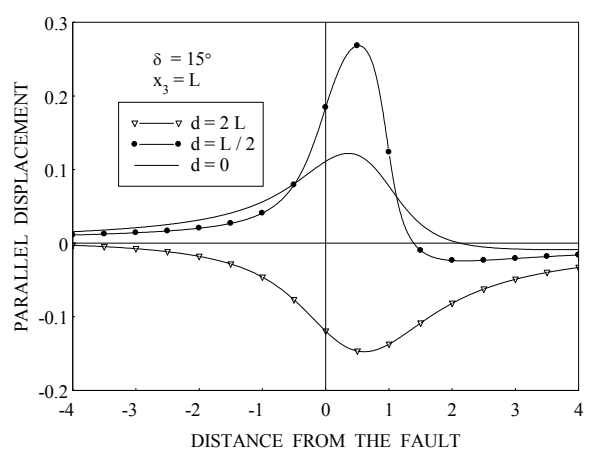

(b)

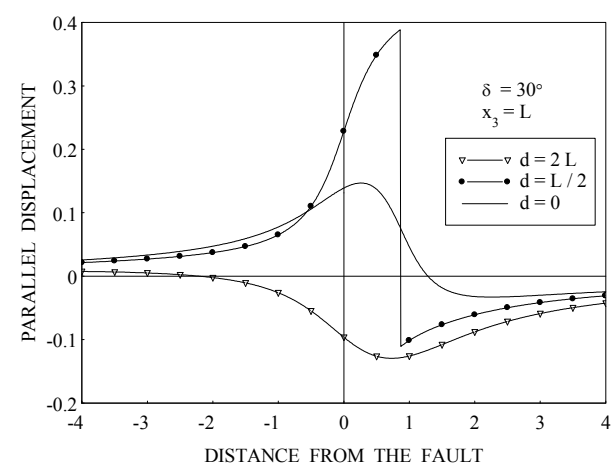

(c)

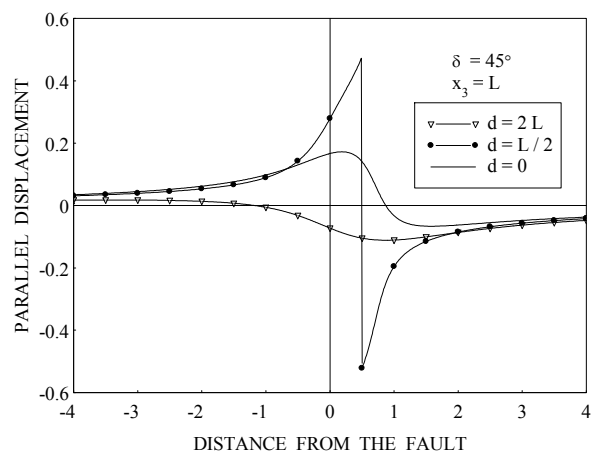

(d)

Figure 3. Variation of the dimensionless horizontal displacement $u_{1}^{(2)} / b$ with distance from the fault $\left(x_{2} / L\right)$ for $x_{3}=L$ for various values of depth $\mathrm{d}$ from the upper edge of the fault for (a) $\delta=0^{\circ}$ (b) $\delta=15^{\circ}$ (c) $\delta=30^{\circ}$ (d) $\delta=45^{\circ}$.

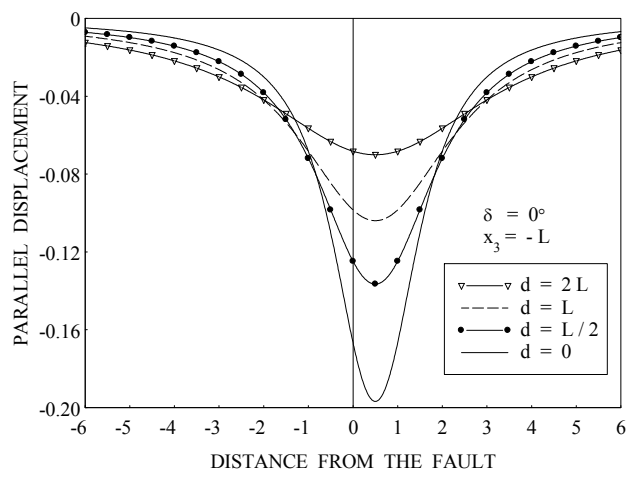

(a)

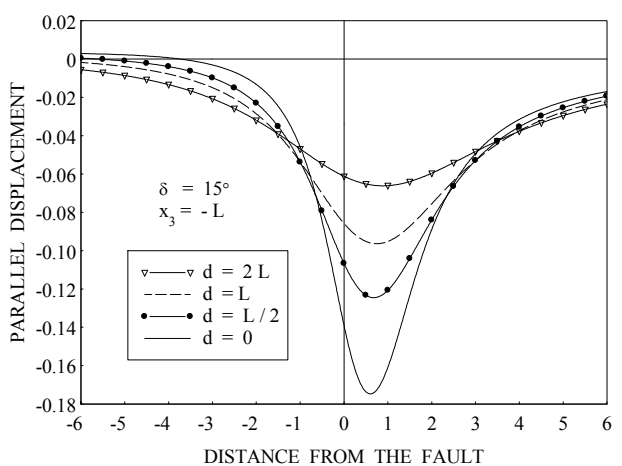

(b)

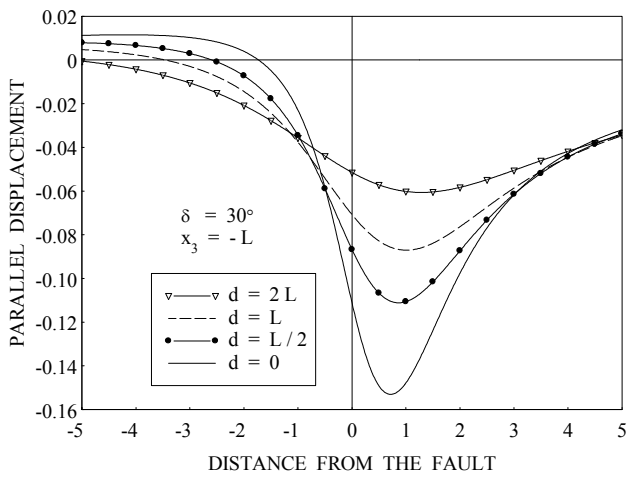

(c)

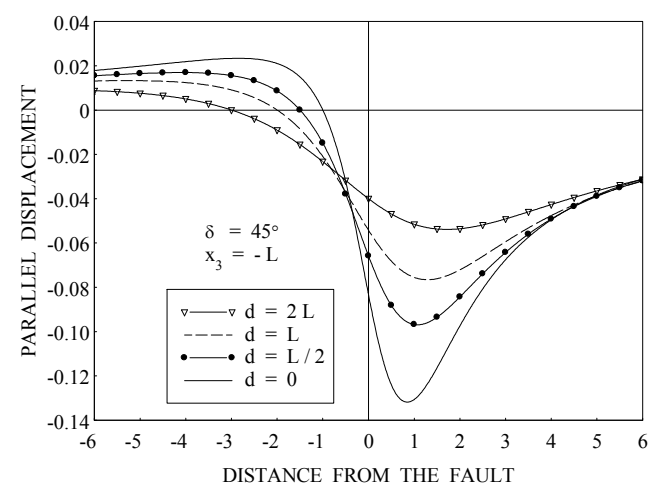

(d)

Figure 4. Variation of the parallel displacement $u_{1}^{(1)} / b$ with $\left(x_{2} / L\right)$ for $x_{3}=-L$ for different values of depth d for (a) $\delta=0^{\circ}$ (b) $\delta=15^{\circ}$ (c) $\delta=$ $30^{\circ}$ (d) $\delta=45^{\circ}$. 


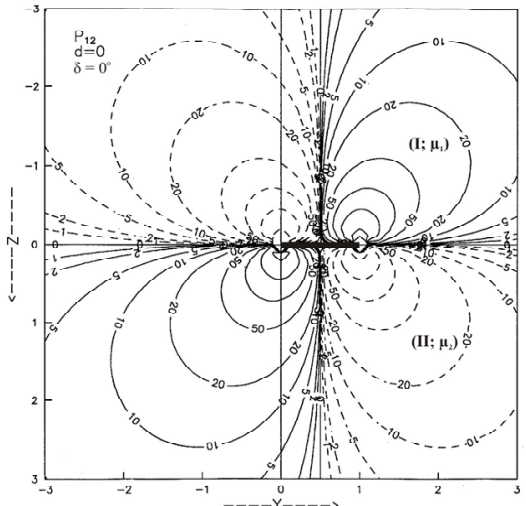

(a)

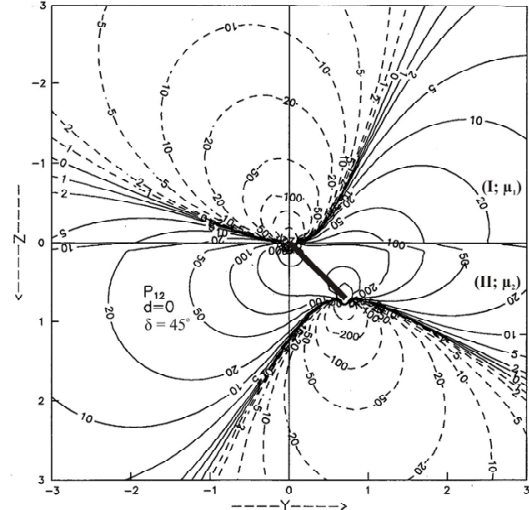

(b)

Figure 5. Contour map for the stress component $p_{12}^{(i)} /\left[\mu_{2} b / L\right],(i=1,2)$ for $\mu_{1} / \mu_{2}=1 / 2$ for interface breaking fault for (a) $\delta=0^{\circ}$ (b) $\delta=45^{\circ}$. Solid lines indicate positive values and dashed lines indicate negative values. The values are in units of $10^{3} \times p_{12}^{(i)} /\left[\mu_{2} b / L\right]$.

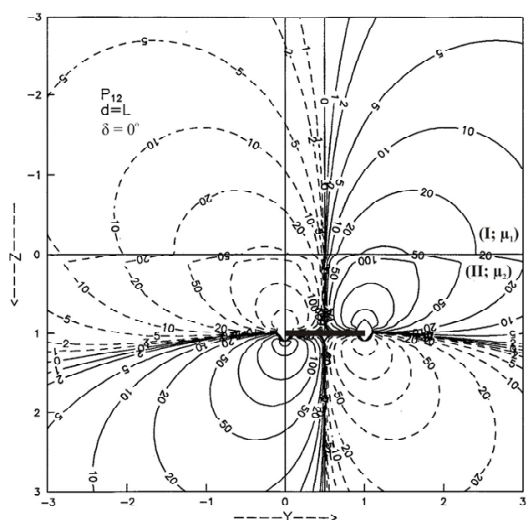

(a)

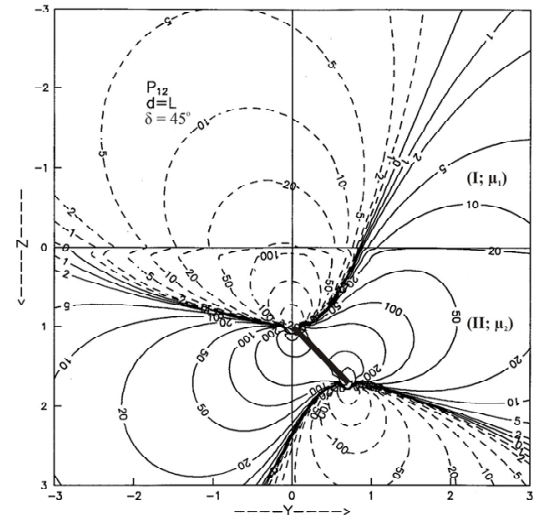

(b)

Figure 6. Contour map for the stress component $p_{12}^{(i)} /\left[\mu_{2} b / L\right], \quad(i=1,2)$ for $\mu_{1} / \mu_{2}=1 / 2$ for buried fault $d=L$ for (a) $\delta=0^{\circ}$ (b) $\delta=45^{\circ}$. Solid lines indicate positive values and dashed lines indicate negative values. The values are in units of $10^{3} \times p_{12}^{(i)} /\left[\mu_{2} b / L\right]$.

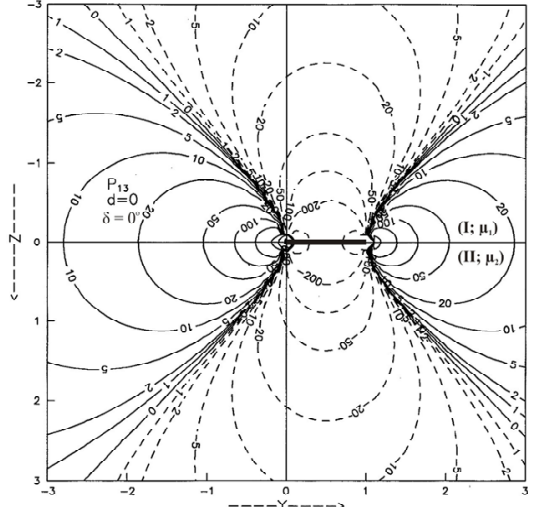

(a)

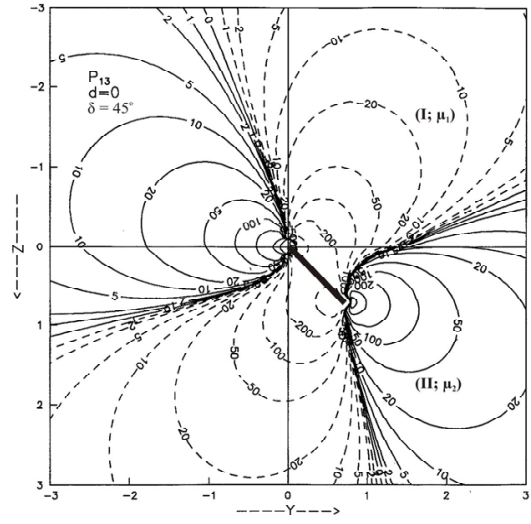

(b)

Figure 7. Contour map for the stress component $p_{13}^{(i)} /\left[\mu_{2} b / L\right],(i=1,2)$ for $\mu_{1} / \mu_{2}=1 / 2$ for interface breaking fault for (a) $\delta=0^{\circ}$ (b) $\delta=45^{\circ}$. Solid lines indicate positive values and dashed lines indicate negative values. The values are in units of $10^{3} \times p_{13}^{(i)} /\left[\mu_{2} b / L\right]$. 


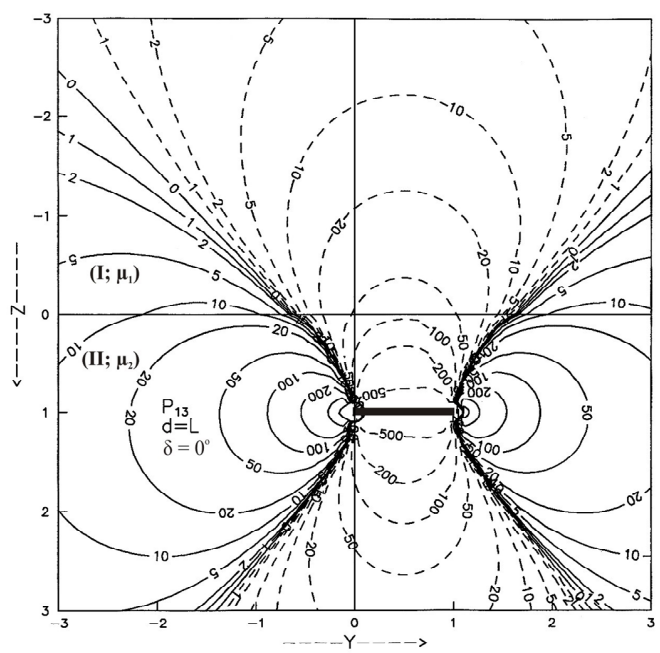

(a)

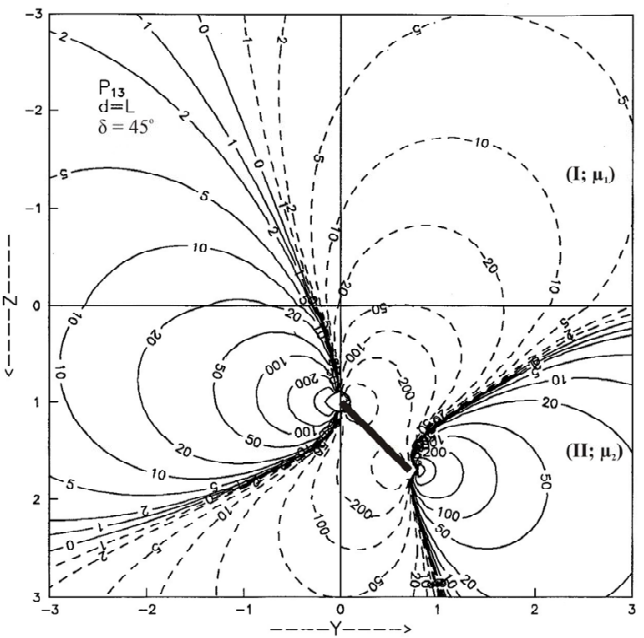

(b)

Figure 8. Contour map for the stress component $p_{13}^{(i)} /\left[\mu_{2} b / L\right], \quad(i=1,2)$ for $\mu_{1} / \mu_{2}=1 / 2$ for buried fault $d=L$ for (a) $\delta$ $=0^{\circ}$ (b) $\delta=45^{\circ}$. Solid lines indicate positive values and dashed lines indicate negative values. The values are in units of $10^{3} \times p_{13}^{(i)} /\left[\mu_{2} b / L\right]$.

for obtaining the deformation due to an inclined strikeslip fault located at an arbitrary depth and arbitrary dip angles. In the earlier paper [4], the results are obtained for $d=0$. Therefore, for small dip angles, the fault approaches near the interface. In the present paper, the depth $d$ is taken explicitly. The effect of variation in depth for a fixed dip and vice-versa can be studied independently.

\section{ACKNOWLEDGEMENTS}

One of the authors SR is thankful to University Grant Commission, New Delhi for financial support in the form of Major Research Project.

\section{REFERENCES}

[1] Singh, S.J. (1985) Static deformation of a multilayered half-space by two-dimensional sources. Acta Geophysica Polonica, 33, 123-134.

[2] Singh, S.J. and Garg, N.R. (1985) On two-dimensional elastic dislocations in a multilayered half-space. Physics of the Earth and Planetary Interiors, 40, 135-145. http://dx.doi.org/10.1016/0031-9201(85)90067-6

[3] Sharma, R.K., Garg, N.R. and Singh, K. (1991) Static deformation of two welded half-spaces due to very long strike-slip dislocations. Proceedings of the Indian Academy of Science (Earth and Planetary Science), 100, 379388.

[4] Rani, S. and Singh, S.J. (1993) Residual elastic field in two welded half-space caused by a long inclined strikeslip fault. Proceedings of the Indian National Science Academy, 59, 455-464.
[5] Rani, S. and Singh, S.J. (2005) A note on 2-D lithospheric deformation due to a blind strike-slip fault. Journal of Earth System Science, 114, 105-110. http://dx.doi.org/10.1007/BF02702013

[6] Chugh, S., Singh, K. and Madan, D.K. (2009) Two-dimensional static deformation of an orthotropic elastic layered half-space due to blind strike-slip fault. ISET Journal of Earthquake Technology, 46, 109-124.

[7] Rybicki, K. (1971) The elastic residual field of a very long strike-slip fault in the presence of discontinuity. Bulletin of the Seismological Society of America, 61, 7992.

[8] Chinnery, M.A. and Jovanovich, D.B. (1972) Effect of Earth layering on earthquake displacement fields. Bulletin of the Seismological Society of America, 62, 1629-1639.

[9] Rybicki, K. (1973) Static deformation of a multilayered half-space by a very long strike-slip fault. Pure and Applied Geophysics, 110, 1955-1966. http://dx.doi.org/10.1007/BF00876559

[10] Rybicki, K. (1978) Static deformation of a laterally inhomogeneous half-space by a two-dimensional strike-slip fault. Journal of Physics of the Earth, 26, 351-366. http://dx.doi.org/10.4294/jpe1952.26.351

[11] Mahrer, K.D. and Nur, A. (1979) Static strike-slip faulting in a horizontally varying crust. Bulletin of the Seismological Society of America, 69, 975-1009.

[12] Mahrer, K.D. and Nur, A. (1979) Strike-slip faulting in a downward varying crust. Journal of Geophysical Research, 84, 2296-2302. http://dx.doi.org/10.1029/JB084iB05p02296 